Hammond A, Prior Y, Horton M, Tennant A, Tyson S (2014). Development of the Evaluation of Daily Activities Questionnaire in Musculoskeletal Conditions: Psychometric Testing. Annals of the Rheumatic Diseases 73 (Suppl 2): 93 doi:10.1136/annrheumdis-2014-eular.3334

\title{
DEVELOPMENT OF THE EVALUATION OF DAILY ACTIVITIES QUESTIONNAIRE IN MUSCULOSKELETAL CONDITIONS: PSYCHOMETRIC TESTING
}

\author{
Alison Hammond ${ }^{1,}{ }^{*}$ Yeliz Prior ${ }^{1}$ Michael Horton ${ }^{2}$ Alan Tennant ${ }^{2}$ Sarah Tyson ${ }^{3}$ \\ ${ }^{1}$ Health Sciences Research, UNIVERSITY OF SALFORD, Salford, ${ }^{2}$ Rehabilitation Medicine, University of Leeds, Leeds, \\ ${ }^{3}$ Nursing, Midwifery \& Social Work, University of Manchester, Manchester, United Kingdom
}

Background: The English Evaluation of Daily Activity Questionnaire (EDAQ) is a patient reported measure of activity limitations including 138 activities in 14 domains. It has been linguistically and culturally validated from the original Swedish to English. In rheumatoid arthritis: confirmatory factor analysis and Rasch analysis have confirmed its internal construct validity, unidimensionality and total scores can be summed in each domain; 12 domains can be included in two components; all domains demonstrate concurrent and discriminative validity, and reliability.

Objectives: To test the validity and reliability of the EDAQ in people with one of seven musculoskeletal conditions (MSCs) in the UK: ankylosing spondylitis (AS); osteoarthritis (OA); systemic lupus (SLE); systemic sclerosis (SS); chronic pain (CP), chronic upper limb conditions (CUL); and Primary Sjogren's (PSS).

Methods: Participants from 20 Rheumatology clinics completed postal questionnaires of demographics, the EDAQ, HAQ, SF36v2, and severity of current condition. Three weeks later, the EDAQ was repeated to evaluate test-retest reliability. Rasch analysis was used to confirm domain and component structure. Cronbach's alpha evaluated internal consistency. Spearman's correlations assessed validity of the EDAQ domains against the other measures and reliability.

Results: 1205 people participated (AS $n=165 ; O A n=184$; SLE $n=164 ;$ SS $n=170 ;$ CP $n=194 ;$ CUL $n=157 ;$ PSS $n=171$ ); $942(78 \%)$ were women; age $=56.61$ years $(S D$ 13.84); condition duration $=17.58$ years $($ SD 14.45$) ; 457(38 \%)$ were employed; 172 (14\%) had dependent children; average pain score was 4.98 (SD 3.70) and fatigue was 5.88 (SD 2.80). Rasch analysis confirmed the 12-domain, 2 component structure in all MSCs.For most conditions, most EDAQ domain scores correlated strongly with HAQ20 ( $r s=0.7-0.92)$ and at least moderately with SF36v2 Physical Functions ( $r s=-0.0 .5$ $--0.91)$, except in the Caring domain which were weak ( $r s=-0.23-0.30)$ as it was applicable to few respondents. Internal consistency was good to excellent for all domains in all conditions, ( $\alpha=0.82$ to 0.98$)$ meaning the EDAQ can be used for groups and individuals. Test-retest reliability $(n=946,79 \%)$ was strong for most domains ( $r s=0.7-0.91)$ except the Caring was moderate ( $r s=0.48-0.64$ ). Although significant and acceptable, values were lower in CULs than other conditions. All EDAQ domains demonstrated significant $(p<0.001)$ discriminant validity, apart from Caring $(p=0.2$ to 0.01$)$.

Conclusions: The English EDAQ is a uni-dimensional, valid, reliable, sensitive measure of daily activity in people with AS, OA, SLE, SS, CP, CUL and PSS. It can therefore be used in both clinical practice and research.

Acknowledgements: This study was funded by the United Kingdom Occupational Therapy Research Fund.

Disclosure of Interest: None declared 